Military Technical College Kobry Elkobbah, Cairo, Egypt

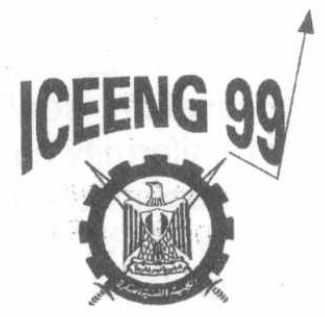

$2^{\text {nd }}$ International Conference on Electrical Engineering ICEENG 99

\title{
SIGNAL TO INTERFERENCE RATIO IN GLOBALSTAR LEO SYSTEM
}

* Abd El-Aziz El-Basioni **Hebat Allah Murad "** Sherief Samy Emam

\section{ABSTRACT}

We consider one LEO CDMA satellite system that consists of 48 satellites running in 8 orbits, each contains 6 satellites. The system is at height $1400 \mathrm{Km}$ from the Earth surface. The performance criteria are the Signal-to-Interference ratio (SIR). Both Dense Traffic Satellite (DTS) and Sparse Traffic Satellite (STS) are considered.

\section{KEYWORDS}

Low Earth Orbit (LEO) Satellite Communication.

* Lucent Technologies, Cairo Office.

** Professor, Dept. of Communication, Faulty of Engineering, Cairo University, Egypt.

${ }^{* * *}$ M. Sc, Dept. of Communication Military technical college. 


\section{INTRODUCTION}

This paper investigates the performance of a LEO satellite system for general CDMAsystem designed for voice communication services. We will show that when CDMA is applied on the uplinks, traffic nonuniformity causes large differences in the signal qualities at succeeding satellites; a satellite above a heavily loaded (dense) traffic area has a low signal- to-interference (SIR), while its neighbor satellites over lightly loaded (sparse) traffic areas have a high level of SIR. The mathematical model for the traffic nonuniformity and the calculations on SIR with a number of numerical examples are given in section II. Through the examples given in section III, we find the situation in which the traffic nonuniformity has the largest effect on the performance of the system and name it the worst case. We propose a traffic assignment control method, which equalizes the traffic loads of service areas to some degree, and investigate its capability with numerical examples in section IV. It is shown that the performance for each user varies according to the user's loc:ation and that the larger possible number of simultaneous transmissions is necessary when nonuniformity of the geographical distribution of the traffic exists.

\section{THE SYSTEM MODEL}

Because of small coverage area of LEO Satellites, compared with geostationary ones, for a global communication network it is necessary to organize the L.EO satellites on a multiple orbit configuration. In this model[1], an area on the Earth is represented by an arc as shown in Fig. 1. In this figure, we distinguish between the coverage area of a satellite and the interference area of it. The coverage area is specified by the minimum value of the elevation angle, $\theta$ min that an Earth station is assumed to be able to access to the satellite while the interference area is determined by the final line of sight of that satellite. The service area is defined as a limited area within a coverage area where users can connect to satellite. The double coverage area is an area commonly located between two or more adjacent coverage areas. It should be noted that if an earth station lies in the interference area but out of the coverage area of a satellite, it would not be allowed to connect to that satellite, but still its signal reaches that satellite as interference. In order to analyz'e the influence of the non-uniformity in traffic, we focus on a series of three satellites and their users. Assume that the total numbers of users, Nu, are distributed randomly in a given area, the size of which is equal to the service areas of three adjacent satellites and their interference areas. For a LEO satellite system in which the satellites have the altitude $\mathrm{h}(\mathrm{km})$, the geometric interference limit for each of them in a srnooth spherical earth assumption is given by the angle.

$$
\beta_{I}=\cos ^{-1}\left(\frac{R}{R+h}\right)
$$

Where $\mathrm{R}$ is the average radius of the earth. The distribution of the users is assumed to have the following function[1,2]: 


$$
P(\alpha)=\left\{\begin{array}{c}
\frac{A}{\omega} \exp \left(-\alpha^{2} / 2 \omega^{2}\right), \\
0
\end{array}\right.
$$

$-\pi \leq \alpha \leq \pi$

elsewhere

Where $\alpha$ is the relative location of a user, measured by the angle at the center of the earth; $\omega$ is the parameter representing uniformity of traffic; $N_{s}$ is the number of satellites in each orbit; and $\mathrm{A}$ is a factor related to the total traffic load (total number of users) in the observed area, the total traffic load for the satellites under consideration should be kept fixed when $\omega$ or $\mathrm{N}_{\mathrm{s}}$, changes. For that purpose, we assume that the total traffic load of three natural service areas, when the peak of the traffic is located at the origin, is constant and equal to $B$ and thus

$$
A=B / \int_{-3 \frac{\pi}{\mathrm{Ns}}}^{3 \frac{\pi}{\mathrm{Ns}}} \exp \left(-\alpha^{2} / 2 \omega^{2}\right) d \alpha
$$

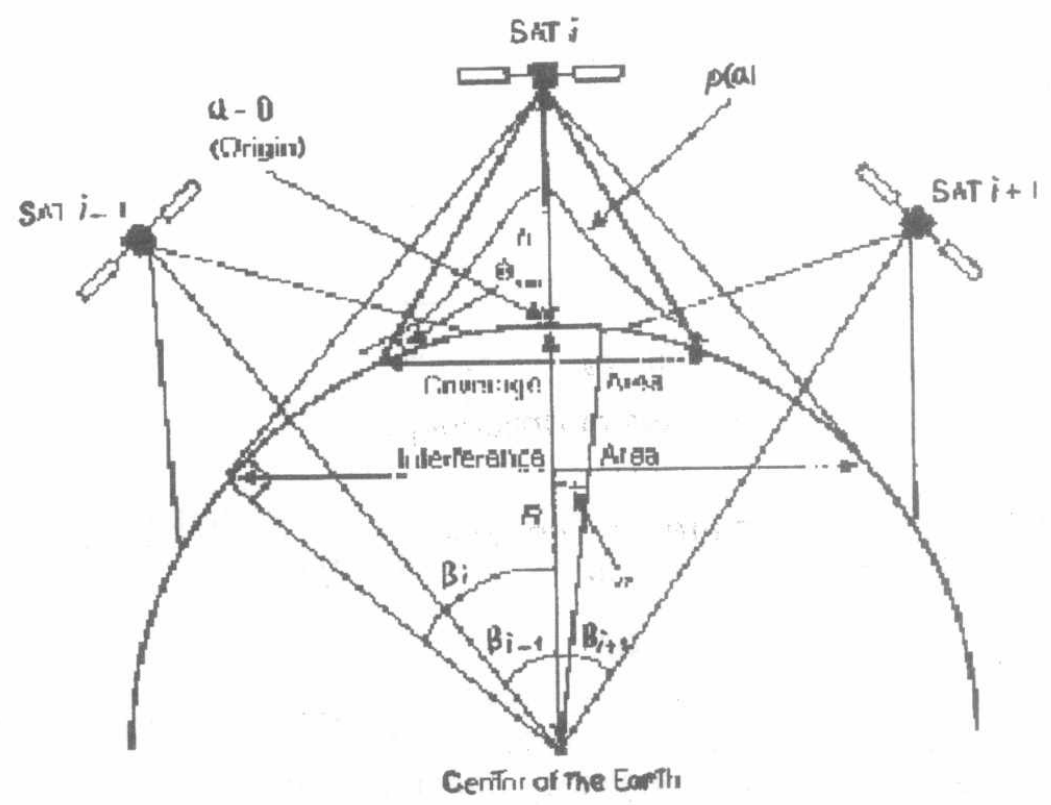

Fig.1. Typical shape of the normal non-uniform traffic model used in analysis

\section{A. Traffic Ratio}

The ratio of the traffic loads of two adjacent natural service areas, when the peak of the traffic is located at the origin, as shown in Fig.2, is defined as: 


$$
T R=\int_{-\pi}^{\pi / N s} P(\alpha) d \alpha / \int_{\pi / N s}^{3 \pi} P(\alpha) d \alpha
$$

We calculate in case of $\omega=0.5, N_{s}=6$, the average number of users of DTS is equal to 70 , the average number of users of STS is equal to 15 , the traffic ratio is nearly equal to 4.7 .

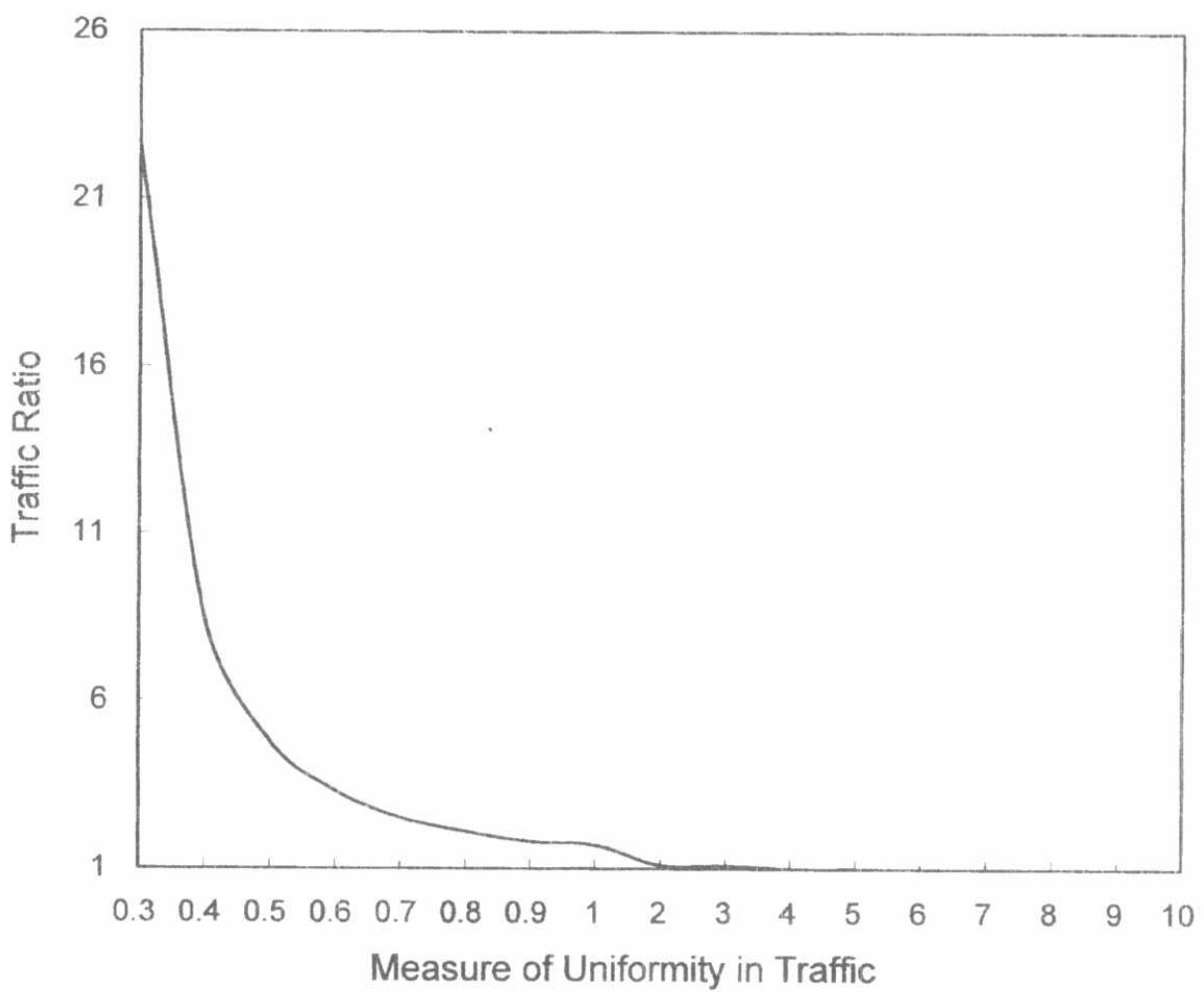

Fig.2 Ratio of traffic in the service area of two adjacent satellites (the first one is over the traffic peak) for different number of satellites in one orbit.

\section{B. The Effect of Satellite Position on SIR}

Because in a LEO satellite system the satellites are on nongeostationay orbits, they are in continuous motion, with relatively high ground speed, which is determined according to the altitude of the satellites, assuming that a non-uniform traffic distribution. Here we examine the changes in SIR characteristics according to the travel of satellites. In this analysis, we assume that the satellites are on circular orbits. the circular orbits usually are used in LEO satellite system. Constellations and simplify the control of the system considerably. 


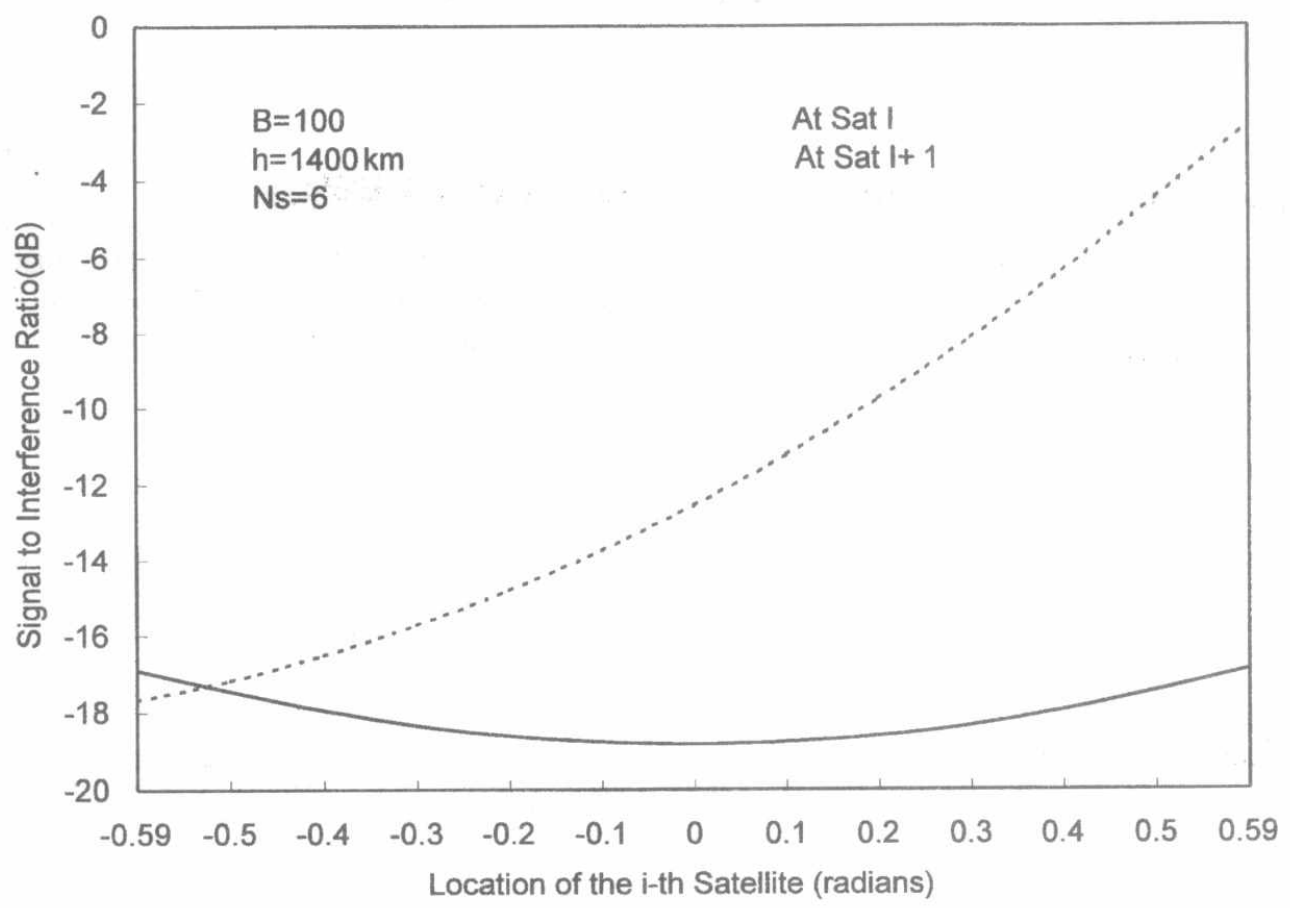

Fig. 3 Changes in SIR characteristics of two neighboring satellites as a function of their angular locations.

Fig. 3 shows the changes in the SIR characteristics of two adjacent satellites, the ith and the $(i+1)$ st ones, as a function of $B_{i}$, that is, the angular position of the ith satellite, for both typical constellation parameters of $h=1400 \mathrm{~km}, \mathrm{~N}_{\mathrm{s}}=6$, and $\omega=0.5$, when $S_{i}=S$ for all satellites, since $B_{i}$ can be determined from

$$
I_{i}=S \int_{i=-\pi / N s}^{\beta+\pi / N s} P(\alpha) d \alpha+S \int_{i-1} \int_{\boldsymbol{\beta}-\boldsymbol{\mu}}^{\boldsymbol{\beta}-\pi / N s} P(\alpha) I_{i-1}^{2}(\alpha) I_{i}^{-2}(\alpha) d \alpha+S \int_{i+1} \int_{\boldsymbol{\beta}+\pi / N s}^{\beta+\beta t} P(\alpha) I_{i+1}^{2}(\alpha) I_{i}^{-2}(\alpha) d \alpha
$$

Assuming counterclockwise rotation of the satellites, $B_{i}=-2 \pi / N_{s}$ is the instant when the ith satellite is far from the peak of the traffic by equal angle as the separation of the satellites, $2 \pi / N_{s}$, and the (i+1)st one is just over this peak, They rotate in their circular orbit with a constant angular velocity, until the ith satellite reaches a symmetrical position to the traffic peak as the start point, that is, $\beta_{i}=2 \pi / N_{s}$. At the halfway point, when $B_{i}=0$, the ith satellite is located just above the traffic peak. If we define the worst situation of system performance as the case when the signal quality at a satellite has the lowest value, from this calculation, this is the case where the peak of the traffic load lies just under one of the satellites, that is $B_{i}=0$ and $B_{i}=-$ $2 \pi / N_{\text {s. }}$ Fig. 3 shows that at those points the satellite above traffic peak had low signal quality ; however, its neighbor satellite has a large value of SIR.An important point drawn from Fig. 3 is that there are large variations in signal quality at each satellite when a nonuniform distribution of users exists. That phenomenon may be acceptable while the level of SIR is higher than a threshold that ensures an acceptable error rate; however, it means that the users of the communications 
system have to accept large tolerances in their service quality performance, even during short periods of time, which is not good behavior for a reliable communications system.

\section{SIR AND TRAFFIC NONUNIFORMITY ANALYTICAL RESULTS}

Here we examine how the degree of traffic nonuniformity affects the performance of the system. In the case where one satellite, say, the ith one, is above the traffic peak, SIR at the ith satellite and its neighbor, the $(i+1)$ st satellite, become $[3,4,5]$.

$$
\begin{gathered}
S_{i}=1 / 2\left[\int_{0}^{\pi / N_{s}} P(\alpha) d \alpha+\int_{\pi / N s}^{\beta I} P(\alpha) \cdot I_{i+1}^{2}(\alpha) \cdot I_{i}^{-2}(\alpha) d \alpha\right] \\
\underbrace{}_{i+1}=y_{2}\left[\int_{\pi / N s}^{3 \pi / N s} P(\alpha) d \alpha+\int_{2 \pi / N s-\theta}^{\pi / N s} P(\alpha) I_{i}^{2}(\alpha) I_{i+1}^{-2}(\alpha) d \alpha+\int_{3 \pi / N s}^{2 \pi / N s+\theta} P(\alpha) I_{i+2}^{2}(\alpha) I_{i+1}^{-2}(\alpha) d \alpha\right]
\end{gathered}
$$

again with the assumption that the satellites have the same designed receiving power levels, S. For the sake of simplicity, let us name the ith satellite above the peak of the traffic with a large number of users as the dense traffic satellite DTS and its neighbors with smaller number of users as sparse traffic satellite STSs. Fig.4 shows the SIR characteristics at the DTS and each STS as a function of traffic nonuniformity for $h=1400 \mathrm{~km}$ and $N_{s}=6$, As can be seen in the figure, in large traffic nonuniformity (i.e., small $\omega$ ), there are large differences between the signal qualities at the satellites, one above the dense traffic area and another above the area with sparse traffic. We see that these differences are large, this is because the traffic ratio between DTS and STS is nearly equal to 1.8. When the number of satellites in each orbit is increased, the service area and hence the number of users connecting to each satellite is decreased. 


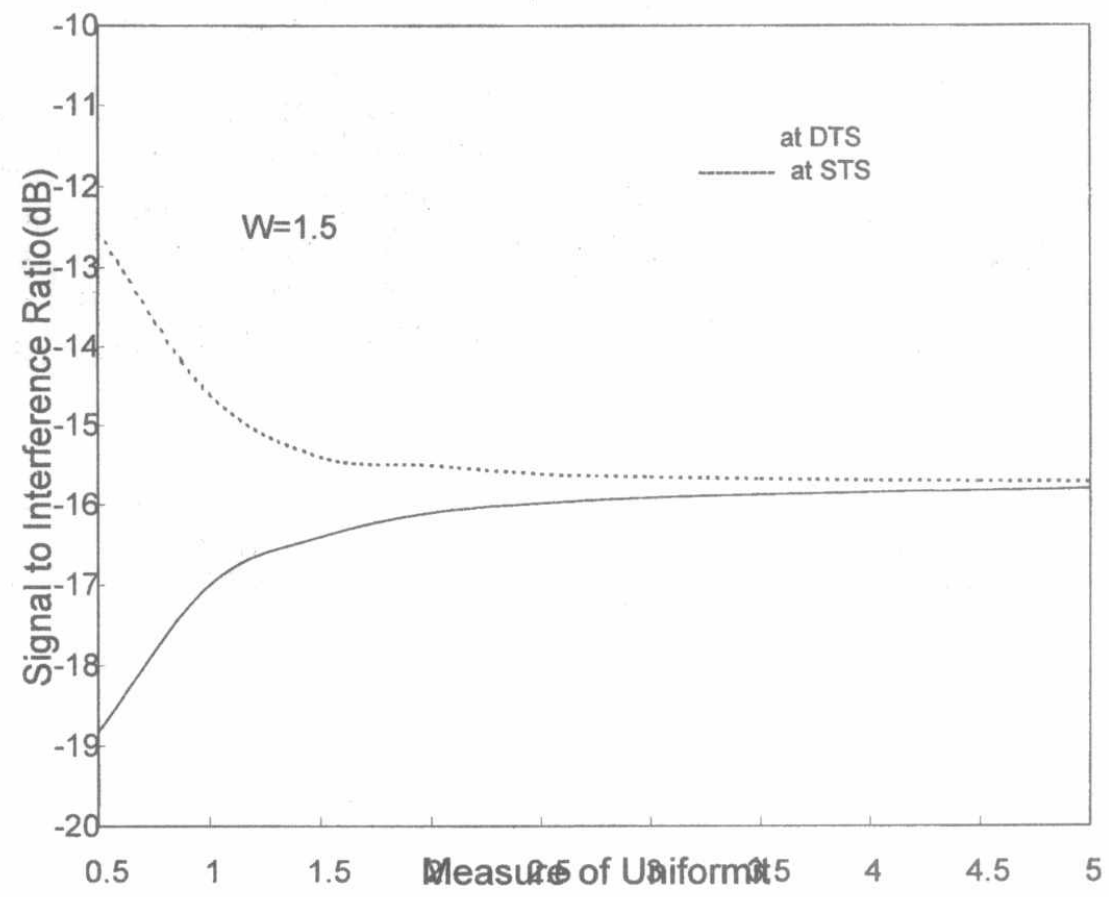

Fig.4 SIR characteristics at DTS and STS with the same required transmitting power levels.

\section{Traffic Assignment Control}

\section{A. Optimum Control}

Since we assumed that the required receiving powers of all satellites are the same and, hence, that service areas of all satellites are equal in size, referred to as natural service area configuration. That configuration, although natural in the case of uniform traffic, no longer has merit when the nonuniform distribution of users is involved. There needs to be a method that can change the size of service areas according to the offered traffic loads. As an example of such a method, this section proposes scheme in which the designed receiving powers of the satellites are not equal. The proposed method would control the size of service areas according to their local traffic loads; that is, the service areas with light traffic loads are expanded, and the ones with heavier traffic loads are decreased.

Let us first assume that the peak of the traffic is located under the ith satellite, that is, the DTS. Because the users communicate with the satellite that needs the smallest transmitting power, by increasing the required transmitting power of the DTS compared with its adjacent satellites on both sides(The STSs), it is possible to increase the tendency of the users in a double coverage area to connect to the STS, not to the DTS, thus decreasing the traffic load of DTS. This method is realized by changing the ratio of designed receiving power of the DTS to that of its neighbors on both sides, say, increasing the ratio $=S_{i} / S_{i-1}=S_{i} / S_{i+1}$. Each satellite counts the number of its users in given period of time and by the means of intersatellite links, for example, the numbers of users of individual satellite are compared with each other, 
and then the proper ratio of in each area for the next period of time is selected and established. Fig. 5 shows the change in SIR as a function of the ratio in a relatively large traffic nonuniformaity situation of $\omega=1.5$. By increasing the ratio from 1 , the number of users of the DTS and STSs is increased and decreased, respectively. Therefore, the performance of the DTS gradually improves and those of STSS degrade. As the ratio of the designed receiving powers, , increases the SIR curves reach to a cross- point. Increasing the powers ratio more, makes the performances of STSs worse than that of the DTS. If we define the optimum control as one that makes all the satellites have the same SIR characteristics, the cross-point exhibits the optimum control . here is 2.7 .

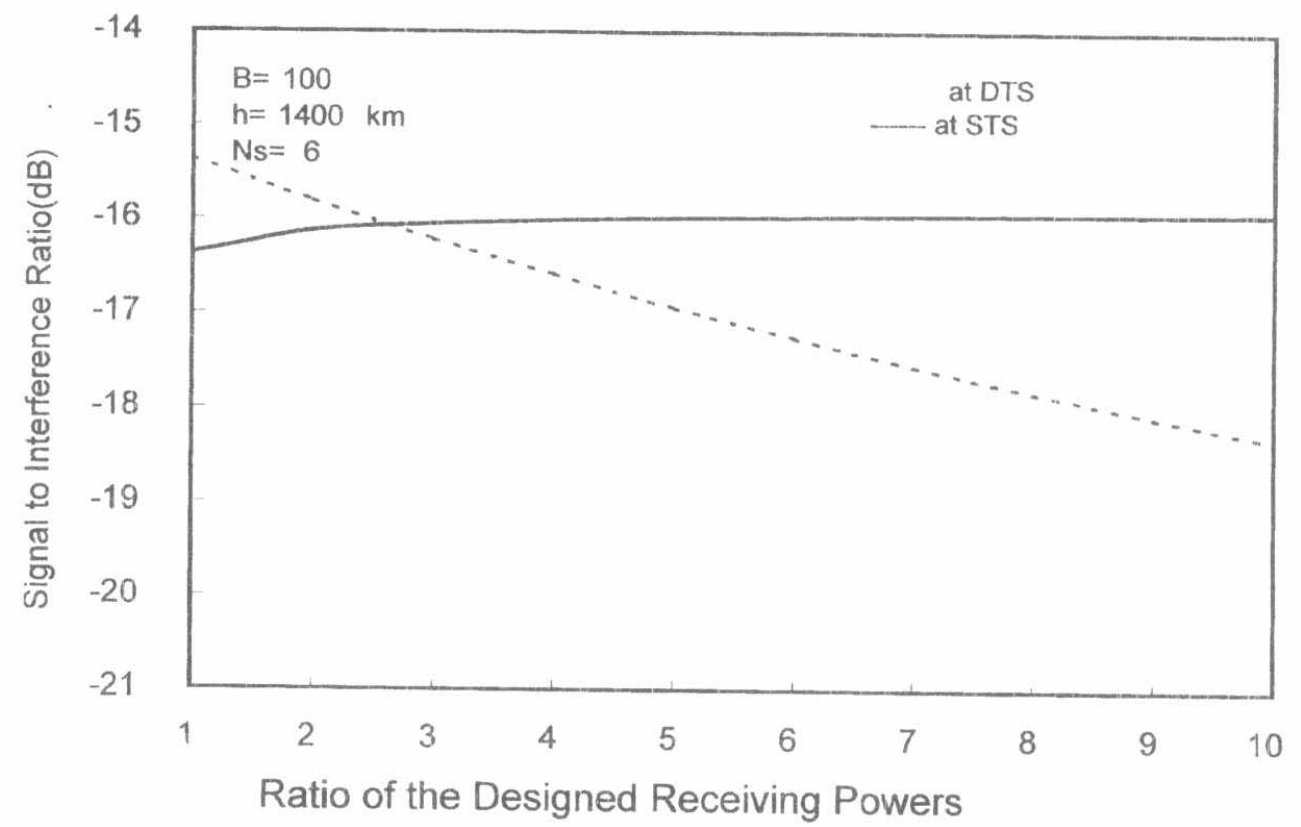

Fig.5 Changes in SIR characteristics at DTS and STS as a result of a change in the ratio of the designed receiving power levels at the satellites.

\section{B. Measuring the Optimum Capability}

Taking appropriate powers ratios that achieve the optimum control for each traffic nonuniformity situation (i.e., for each $\omega$ ) gives the SIR characteristics shown in Fig.6 for $h=1400 \mathrm{~km}, N_{s}=6$. That figure shows the changes in SIR characteristics at DTS and STS before and after applying the control scheme, where the curve of optinum control show the SIR at both the DTS and the STS when optimum control is employed. As shown in Fig.6, we are able to improve the performance of the [DTS, that is, the satellite with heavy traffic load. There are two reasons for that improvement. First, by increasing the required transmitting power of the DTS, the service area of the DTS becomes smaller, and thus interference from its own users decreases. Second, lower transmitting powers of the users of STSs make the interference power from their users smaller. The latter occurs for every case, and if the double coverage area becmes larger, the former effect also can be expected. Fig.6 is the effect of controlling the required transmitting powers of satellites in its optimum case, for $\mathrm{Ns}=6$. 


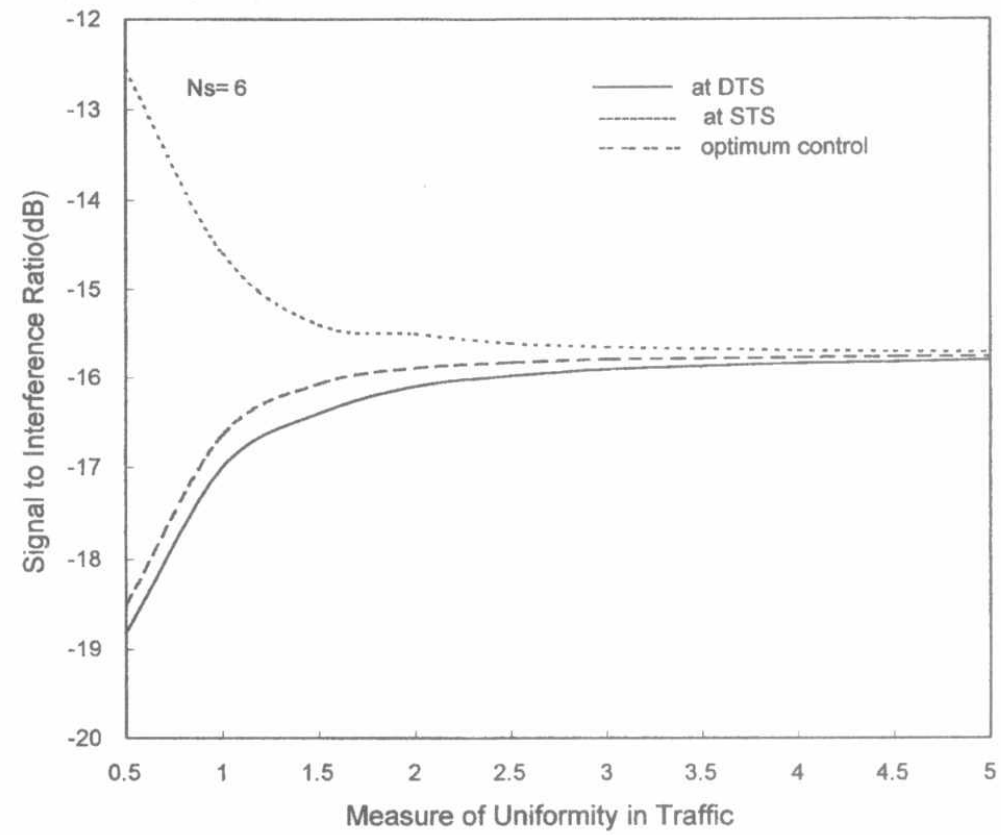

Fig.6 Effect of controlling the required transmitting powers of satellites in its optimum case, for $\mathrm{Ns}=6$.

\section{CONCLUSION}

This paper models the nonuniformity of the traffic loads in a satellite based communication system employing LEO satellites and analyzes the performance of the system. It is shown that the performance of LEO system, measured by the value of SIR at each satellite, degrades as a direct result of nonuniformity in distribution of users. Also it is shown that the worst case in the performance of the satellite system happens when the peak of traffic load lies just under one of the satellite. In addition, a modified power control method which changes the required transmitting power levels, according to the traffic load in coverage area of satellites, is examined and is shown that this method can remedy the performance degradation of the system to some degrees.

\section{REFERENCES}

[1] A. Jamalipour et al., "performance of an integrated voice/Data system in non-uniform traffic low earth orbit satellite communication systems, "IEEEJ. Select. Areas Commun., Vol. 13, No. 2, 1995, PP. 465-473.

[2] A.Jamalipour, M. Katayama, T. Yamazato, and A. Ogawa,"Signal-tointereference of CDMA in low earth orbital satellite communication systems with nonuniform traffic distribution " in Proc. IEEE GLOBE-COM'94, San Francisco, CA, 1994, PP. 1748-1752.

[3] Pritchard, W. L., H. G. Suyderhoud, and R. A. Nelson, Satellite Communication Engineering, $2^{\text {nd }}$ ed, Englewood Cliffs, NJ: Prentice-Hall, 1987.

[4] Roddy, D., Satellite Communications,Englewood Cliffs, NJ: Prentice-Hall,1989. 
Proceedings of the $2^{\text {nd }}$ ICEENG Conference, 23-25 Nov. 1999

\begin{tabular}{l|l}
$\mathrm{AC}-1$ & $10]$ \\
\hline
\end{tabular}

[5] Werner, M., et al., "Analysis of System Parameters for LEO/ICO-Satellite Communication Networks, " IEEE J. Select. Areas Commun., Vol. 13, No. 2 , 1995, pp.371-381. 